\title{
ON ROTATION GROUPS OF PLANE CONTINUOUS CURVES UNDER POINTWISE PERIODIC HOMEOMORPHISMS
}

\author{
DICK WICK HALL
}

In this paper we make use of the work of G. T. Whyburn ${ }^{1}$ on light interior transformations and on orbit decompositions of certain spaces to obtain a theorem by means of which a certain subset of the orbits of points under a periodic transformation $T(M)=M$ may be given a linear ordering. This theorem is then used to obtain an accessibility theorem for plane continuous curves similar to one previously published by $\mathrm{L}$. Whyburn. ${ }^{2}$ We take this opportunity to express our indebtedness to G. E. Schweigert for suggesting the proof of Theorem I given below and thus eliminating the longer and less interesting proof previously obtained by the author. For any $x \in M$, the orbit of $x$ un$\operatorname{der} T$ means $O(x)=\sum_{i=-\infty}^{\infty} T^{i}(x)$.

THEOREM I. Let $M$ be a locally connected continuum (that is, a continuous curve) and $T(M)=M$ an arbitrary periodic homeomorphism. Then if $a$ and $b$ are arbitrary points of $M$ lying in different orbits under $T$ and if $a x b$ is any simple arc in $M$ joining $a$ and $b$, then there must exist a simple arc $a^{\prime} x^{\prime} b^{\prime}$ in $M$ lying in the orbit of axb under $T$ such that $a^{\prime}$ belongs to $O(a), b^{\prime}$ belongs to $O(b)$ and no two points of $a^{\prime} x^{\prime} b^{\prime}$ lie in the same orbit under $T$. Furthermore, the point $a^{\prime}$ may be any arbitrary preassigned point of the orbit of $a$.

Proof (Schweigert). Let $M^{\prime}$ be the hyperspace obtained by decomposing the space $M$ into its orbits under $T$. Then, since the orbit decomposition is continuous, ${ }^{8}$ it follows ${ }^{4}$ that there exists a light interior transformation $f(M)=M^{\prime}$, namely, the transformation given by and associated with the orbit decomposition. Let $a x b$ be the given arc in $M$. Then we may assume without loss of generality that $a x b$ has precisely the point $a$ in common with $O(a)$ and precisely the point $b$ in common with $O(b)$. Define $K=f(a x b)$. Then $K$ is a locally connected continuum containing $c=f(a)$ and $d=f(b)$. Let $c y d$ be an arc in $K$ joining $c$ to $d$. Now let $a^{\prime}$ be an arbitrary point of $O(a)$. Then ${ }^{5}$

Presented to the Society, February 26, 1944; received by the editors April 13, 1944.

${ }^{1}$ See G. T. Whyburn, Analytic topology, Amer. Math. Soc. Colloquium Publications, vol. 28, 1942, pp. 182-189 and 239-262.

'See L. Whyburn, Rotation groups about a set of fixed points, Fund. Math. vol. 28 (1937) pp. 124-130, in particular p. 127.

S See G. T. Whyburn, loc. cit. p. 258.

4 See G. T. Whyburn, loc. cit. p. 130.

${ }^{\circ}$ See G. T. Whyburn, loc. cit. p. 186. 
there must exist a simple arc $a^{\prime} x^{\prime} b^{\prime}$ in $M$ such that $f\left(a^{\prime} x^{\prime} b^{\prime}\right)=c y d$ is topological. By definition of $f$ we see that each point of $a^{\prime} x^{\prime} b^{\prime}$ belongs to the orbit of some point of $a x b$, and from the one-to-oneness of this transformation it is immediate that no two points of $a^{\prime} x^{\prime} b^{\prime}$ lie in the same orbit under this transformation. This completes the proof.

CoRollary. The same conclusion holds for any pointwise periodic $T(M)=M$ if we impose the additional restriction either that $T$ have equicontinuous powers ${ }^{6}$ or that the period function remain bounded on the arc $a x b$.

The accessibility theorem for plane continuous curves mentioned in the introductory paragraph of this paper may be stated as follows.

TheOREM A (L. WhybuRN). If $M$ is a plane continuous curve and $T(M)=M$ is a homeomorphism and if $C$ is an element of a rotation group of $M$ under $T$ of order at least two, then $C$ has property $S .{ }^{7}$

The object of our second theorem is to obtain a result similar to Theorem A, but with the emphasis in the hypothesis placed upon the type of the transformation $T$ rather than upon the order of the rotation group under consideration. Before stating the theorem we recall certain important subsets of $M$. By $L$ we denote the closed invariant subset of $M$ consisting of those points at which the period function has an unbounded limit superior. By $K$ we denote the collection of all fixed points of $M$ under $T$. If $R$ is a component of $M-K$, then $R$ is an element of a rotation group under $T$; this rotation group consists exactly of the orbit of $R$ under $T$; and its order is the number of components which it contains. The order of a rotation group under $T$ may, of course, be either finite or infinite.

We are now in a position to state our second theorem.

TheOREM II. Let $M$ be a plane continuous curve and $T(M)=M$ an arbitrary homeomorphism, while $R$ denotes an element of some rotation group of $M$ under $T$. Then

(a) If $K$ is locally connected then $R$ has property $S$ and every point of $F(R)=\bar{R}-R$ is regularly accessible from $R$.

(b) If $T$ is pointwise periodic and has equicontinuous powers we get the same conclusion as in (a).

(c) If $T$ is pointwise periodic then every point of $F(R)$ which is not $a$ point of $L$ is regularly accessible from $R$.

Proof. Note that (a) is immediate from a theorem of G. T. Why-

- See G. T. Whuburn, loc. cit. p. 258.

' See G. T. Whyburn, loc. cit. p. 20.

See G. T. Whyburn, loc. cit. p. 111. 
burn, ${ }^{9}$ and that it also follows at once from the proof of Theorem A.

We give the proofs of (b) and (c) simultaneously, making use of the corollary to Theorem $I$ in each case. It is to be noted that our proof is similar to the original proof of Theorem A.

Denote by $d$ a positive number exceeding the diameter of the set $M$, and suppose that $M$ is embedded in the upper half of the Euclidean plane. If the theorem be false there must exist a point $p$ in $F(R)$ which is not regularly accessible from $R$ and (unless $T$ has equicontinuous powers) such that $p$ does not lie in $L$. This means that there exists a positive number $\epsilon$ and a sequence of points $\left\{p_{i}\right\}$ of $R$ converging to $p$ such that no two points of this sequence may be joined in $R$ by a connected set of diameter less than $17 \epsilon$. Let $C_{p}$ be a circle of radius $8 \epsilon$ having its center at the point $p$. No generality is lost by the following assertion:

(1) For every $i$ the set $O\left(p_{i}\right)$ lies within $C_{p}$; no two of the points $p_{i}$ may be joined by a connected subset of $R$ lying within this circle; and if $T$ does not have equicontinuous powers then there exists an integer $N$ such that no point of $M$ lying within $C_{p}$ has period greater than $N$ under $T$.

For some point $q^{\prime}$ of $R$ exterior to $C_{p}$ we construct arcs $p_{i} q^{\prime}$ in $R$ for every integer $i$ and we denote the first intersection of the $\operatorname{arc} p_{i} q^{\prime}$ with the circle $C_{p}$ by $q_{i}$. It follows from (1) that the arcs $p_{i} q_{i}$ are pairwise disjoint and we may assume, exactly as in the proof of Theorem A, that this sequence of arcs converges to a limiting set $H$ which is a subcontinuum of $K$. Making use of the corollary to Theorem I we can insure that no $\operatorname{arc} p_{i} q_{i}$ meets the orbit of any point of $M$ in more than a single point, and that no two of these arcs meet the orbit of the same point of $M$. This means, in particular, that no two consecutive images under $T$ of any one of these arcs will have a point in common. We may assume that the sequence $\left\{q_{i}\right\}$ converges monotonically on $C_{p}$ to a point $q$.

We place the $x$-axis in such a position that $p$ lies at the point $(-4 \epsilon, 2 d)$ and $q$ at the point $(4 \epsilon, 2 d)$. By $L_{i}(i= \pm 1, \pm 2, \pm 3)$ we denote the line segment joining $(i \epsilon, 0)$ to $(i \epsilon, 4 d)$, and by $D_{i, j}$ the interior of the rectangle formed by $L_{i}, L_{j}, y=0$, and $y=4 d$.

Using the fact that $H$ is a subset of $K$ and either (b) or (c) ${ }^{10}$ we may make the following assumption without loss of generality:

(2) If $x$ is any point of any arc $p_{i} q_{i}$ then $O(x)$ lies within a circle having its center at some point of $H$ and diameter $\epsilon / 2$.

Let $r_{i} s_{i}$ be a subarc of $p_{i} q_{i}$ with its interior in $D_{-3,3}$ and its end

- See G. T. Whyburn, Concerning the open subsets of a plane continuous curve, Proc. Nat. Acad. Sci. U.S.A. vol. 13 (1927) pp. 650-657, in particular Theorems 1 and 5 of this paper.

${ }^{10}$ See G. T. Whyburn, Analytic topology, loc. cit. p. 252. 
points on $L_{-3}$ and $L_{3} ; r_{i}^{\prime} s_{i}^{\prime}$ the $\operatorname{arc} T\left(r_{i} s_{i}\right) ; x_{i}^{\prime} y_{i}^{\prime}$ a subarc of $r_{i}^{\prime} s_{i}^{\prime}$ with its interior in $D_{-2,2}$ and its end points on $L_{-2}$ and $L_{2}$; and $x_{i} y_{i}$ the $\operatorname{arc} T^{-1}\left(x_{i}^{\prime} y_{i}^{\prime}\right)$. The existence of the arc $x_{i}^{\prime} y_{i}^{\prime}$ follows from (2) and by the same token we see that $x_{i} y_{i}$ is a subarc of $r_{i} s_{i}$ having its end points in the respective regions $D_{-3,-1}, D_{1,3}$. Now the sequence of $\operatorname{arcs}\left\{x_{i}^{\prime} y_{i}^{\prime}\right\}$ may be assumed to converge to a subcontinuum $H^{\prime}$ of $H$ which is, of course, disjoint with every one of these arcs. This means that by taking a subsequence the following assumption will hold.

(3) For any fixed integer $i$ and every $k$ exceeding $i$ the arc $x_{k}^{\prime} y_{k}^{\prime}$ separates $D_{-2,2}$ between $x_{i}^{\prime} y_{i}^{\prime}$ and $x_{n}^{\prime} y_{n}^{\prime}$ for every $n$ greater than $k$. Thus $x_{k}^{\prime} y_{k}^{\prime}$ separates $D_{-2,2}$ between $x_{i}^{\prime} y_{i}^{\prime}$ and $H^{\prime}$ for every $k$ exceeding $i$.

For any fixed value of $i$ we know that the closed sets $O\left(x_{i} y_{i}\right)$ and $H^{\prime}$ are disjoint, which means that there will exist a region $U_{i}$ in the plane containing $H^{\prime}$ but disjoint with $O\left(x_{i} y_{i}\right)$. We may assume that $U_{i}$ contains $O\left(x_{k} y_{k}\right)$ for every $k$ exceeding $i$. We also know, in view of this last remark, that for $i$ fixed either $x_{i} y_{i}$ separates $D_{-1,1}$ between $x_{i}^{\prime} y_{i}^{\prime}$ and every $x_{k}^{\prime} y_{k}^{\prime}$ for $k$ exceeding $i$ or $x_{i}^{\prime} y_{i}^{\prime}$ separates this region between $x_{i} y_{i}$ and $x_{k}^{\prime} y_{k}^{\prime}$ for every $k$ exceeding $i$. By taking a subsequence and renumbering we can insure that the same one of these two statements holds for every value of $i$ and thus obtain the following assertion.

(4) If $i$ and $k$ be any two distinct integers then in the region $D_{-1,1}$ the four arcs $x_{i} y_{i}, x_{i}^{\prime} y_{i}^{\prime}, x_{k} y_{k}, x_{k}^{\prime} y_{k}^{\prime}$ must occur either in the order just specified or in the alternative order $x_{i}^{\prime} y_{i}^{\prime}, x_{i} y_{i}, x_{k}^{\prime} y_{k}^{\prime}, x_{k} y_{k}$.

No generality is lost by the assumption that for every integer $i$ the arc $x_{i} y_{i}$ has an interior point within the circle $C_{z}$, having its center at a point $z$ on the $y$-axis and radius sufficiently small so that any two points of $M$ lying within $C_{z}$ may be joined by an arc of $M$ the orbit of which lies within $D_{-1,1}$. This enables us to find a simple $\operatorname{arc} a_{i} b_{k}$ lying in $M$ with $O\left(a_{i} b_{k}\right)$ in $D_{-1,1}$ and having exactly the points $a_{i}, b_{k}$ in common with $x_{i} y_{i}$ and $x_{k} y_{k}$, respectively. As the two arrangements given in (4) are symmetrical we need treat only the case of the first one; the other will follow by a simple interchange of the letters $i$ and $k$. From (1) we see that the arc $a_{i} b_{k}$ must contain at least one point of the closed set $K$, and we denote by $f_{i k}$ the last point of $K$ on this arc. Then if $g_{k}$ be the first point of $O\left(x_{k} y_{k}\right)$ on the arc $f_{i k} b_{k}$, it follows that for some integer $n$ the point $g_{k}^{\prime}=T^{n}\left(g_{k}\right)$ lies on the arc $x_{k}^{\prime} y_{k}^{\prime}$. Thus the $\operatorname{arc} f_{i k} g_{k}^{\prime}=T^{n}\left(f_{i k} g_{k}\right)$ is a simple arc lying in $D_{-1,1}$ and joining the point $f_{i k}$ to a point of $x_{k}^{\prime} y_{k}^{\prime}$, while containing no point of $x_{k} y_{k}$. This contradiction completes the proof of Theorem II.

UNIVERSTTY OF MARYLAND 